\title{
Time-Frequency Packing Applied to Cost-Effective IM/DD Transmission Based on Directly Modulated VCSEL
}

\author{
Antonio Malacarne, Francesco Fresi, Gianluca Meloni, Tommaso Foggi, and Luca Potì, Member, IEEE
}

\begin{abstract}
Potential of directly modulated vertical cavity surface emitting lasers (VCSELs), widely employed for low-cost and energy-efficient intradata center transmissions, is here studied for transmissions up to access/metro scenarios. In particular, the manuscript refers to long-wavelength VCSELs for intensity modulation/direct detection (IM/DD) ON-OFF keying transmission over standard single-mode fiber (SSMF). The intent of this paper primarily consists of demonstrating information rate and fiber reach enhancement in potentially uncooled environments or with relaxed thermal management requirements through the use of timefrequency packing (TFP) technique in a VCSEL-based transmission. TFP has been applied here to IM/DD transmission for the first time, although not in a multiple channel system. The digital signal processing unit implementation is clearly simplified with respect to coherent systems (no frequency synchronization and no phase error recovery), where TFP was firstly introduced, as it only takes into account the photo-detected signal intensity, regardless of the phase information. On the other hand, the signal processing is here more complex than in a traditional IM/DD system. Through the use of a commercial C-band 4 G VCSEL, net information rate up to 12.5 and $11.2 \mathrm{~Gb} / \mathrm{s}$ has been measured for 25 and $45 \mathrm{~km}$ of SSMF, respectively, in an unamplified optical link and with power budget equal to 19 and $16 \mathrm{~dB}$, respectively. The transmission is intrinsically polarization independent and wavelength independent and tolerance to temperature increase up to $60^{\circ} \mathrm{C}$ has been analyzed together with energy consumption.
\end{abstract}

Index Terms-Access networks, intensity modulation/direct detection, metro networks, short reach, time-frequency packing, vertical cavity surface emitting laser.

\section{INTRODUCTION}

$\mathbf{T}$ HE continuously increasing demand for higher performance, lower cost and lower power consumption of transceivers for optical fiber communication systems, gave rise

Manuscript received June 15, 2017; revised July 24, 2017; accepted August 7, 2017. Date of publication August 22, 2017; date of current version September 8, 2017. This work was supported in part by the European FP7 Project "RAPIDO" (G. A. 619806) and in part by the European H2020 Project "ROAM" (G. A. 645361). (Corresponding author: Antonio Malacarne.)

A. Malacarne and F. Fresi are with the Institute of Communication, Information and Perception Technologies, Sant'Anna School of Advanced Studies, Pisa 56124, Italy (e-mail: antonio.malacarne@sssup.it; francesco.fresi@ sssup.it).

G. Meloni, T. Foggi, and L. Potì are with the National Laboratory of Photonic Networks, Consorzio Nazionale Interuniversitario per le Telecomunicazioni, Pisa 56124, Italy (e-mail: gianluca.meloni@cnit.it; tommaso.foggi@cnit.it; luca.poti@cnit.it).

Color versions of one or more of the figures in this paper are available online at http://ieeexplore.iee.org.

Digital Object Identifier 10.1109/JLT.2017.2743529 to the advent of vertical cavity surface emitting lasers (VCSELs), in particular for short reach scenarios where massive low-cost production of energy efficient modules is a must. Indeed, thanks to their vertical structure, tens of thousands of VCSELs can be processed simultaneously on a single wafer. In addition, in contrast to edge-emitting lasers, for the same reason they can be tested at several stages throughout the fabrication process to check for material quality and processing issues, so as to limit waste of time and materials. Furthermore, their suitability to be embedded in integrated modules for optical interconnects and intra-data center scenarios is given by the potential for hybrid integration into Silicon (Si) - based transceivers [1][4] and remarkable electro-optical energy conversion efficiency. Moreover, VCSELs can be driven at low driving currents and operate at high temperatures thus reducing operational costs related to thermal stabilization [5]. The possibility to achieve direct intensity modulation (IM) of VCSEL allows low driving current and consequent low power consumption as well as low-cost direct detection (DD) at the receiver. In IM/DD systems the data rate is limited by the modulation bandwidth that, for long-wavelength (LW)-VCSELs, typically reaches up to $10 \mathrm{GHz}$ for commercial devices [6], [7] and up to $18 \mathrm{GHz}$ for research prototypes [8]. To increase the information rate 4-level pulse amplitude modulation (4-PAM) is often preferred, thus doubling the data rate with a reasonable hardware cost and complexity [9], [10]. However, the large frequency chirp induced by direct modulation of VCSELs in interaction with chromatic dispersion heavily limits the data rate and the fiber reach in transmission systems based on standard single-mode fiber (SSMF) in the $1550 \mathrm{~nm}$ window. This drawback represents the main reason why directly-modulated (DM)-VCSELs are massively employed in short reach scenarios only. However, long-wavelength (LW)-VCSELs with emission in the C-band range, which can benefit from optical amplification, are currently under development due to the possibility to employ novel techniques to extend the fiber reach up to access and metro scenarios. Different approaches have been proposed to mitigate this issue, such as injection locking of VCSEL to another laser [11] or to another VCSEL [12] or the use of electronic dispersion compensation [13]. Another approach to increase the data rate and the fiber reach up to the metro-regional networks needs is the use of coherent detection and/or generation of more complex modulation formats involving optical phase modulation 
too. In this case, VCSELs are employed as continuous wave laser sources only (for both transmitter and local oscillator, LO), the use of external in-phase/quadrature (IQ) modulation is necessary and considerably increases the energy consumption at the transmitter, high-complexity digital signal processing (DSP) at the coherent receiver is needed to compensate for the strong VCSEL phase noise and wavelength tuning of the $\mathrm{LO}$ according to the transmitter emission wavelength is required [14]-[17].

Concerning access networks applications, recent efforts focus on techniques to enhance both the data rate and the fiber reach in case of bandwidth-limited directly-modulated (DM) lasers, still keeping on-off-keying (OOK) modulation, together with wavelength-dependent simplified coherent detection that, however, could hardly be exploited in case of DM-VCSELs due to their phase noise and drift of the emission optical frequency [18].

In this work, which represents and extension of [19], we propose and experimentally demonstrate a novel solution for increasing both the transmission data rate and the optical reach thanks to the so called time-frequency packing (TFP) technique [20]-[22], while preserving simple OOK IM obtained through DM-VCSEL and DD with an avalanche photodiode (APD). Primarily TFP was introduced in coherent optical systems [20], and then applied by the authors in [14] to improve the spectral efficiency (SE) of $125 \mathrm{~Gb} / \mathrm{s}$ dual-polarization quadrature-phase shift keying (DP-QPSK) wavelength division multiplexing (WDM) signal obtained through external IQ modulation for regional networks applications $(\sim 800 \mathrm{~km})$. The same concept has been successfully applied here to an IM/DD system, through a DSP unit which is clearly simpler if compared to coherent systems (no frequency synchronization and no phase error recovery), and which only takes into account the photo-detected signal intensity, regardless of the phase information. In the presented experiment a $14 \mathrm{~Gb} / \mathrm{s}$ gross information rate $(12.5 \mathrm{~Gb} / \mathrm{s}$ net) is achieved with a $4 \mathrm{G}$ commercial VCSEL (by RayCan) and transmission over $25 \mathrm{~km}$ of SSMF is demonstrated at $1550 \mathrm{~nm}$. Indeed, the TFP technique is intrinsically robust to inter-symbol interference (ISI) that, in the considered case, gets generated from both the limited VCSEL modulation bandwidth and the dispersion effect. Thanks to TFP an information rate three times higher than the VCSEL nominal data rate is achieved, dispersion tolerance is improved and $19 \mathrm{~dB}$ power budget without optical amplification is experimentally demonstrated. Additional measures over $45 \mathrm{~km}$ of SSMF demonstrate error-free operation at $11.2 \mathrm{~Gb} / \mathrm{s}$ and $16-\mathrm{dB}$ power budget. The system is intrinsically polarization- and wavelength-independent and requires neither coherent detection nor DSP at the transmitter. As final step the system has been tested with success at $50{ }^{\circ} \mathrm{C}$ and $60{ }^{\circ} \mathrm{C}$ over $25 \mathrm{~km}$ of SSMF, reaching up to $14 \mathrm{~Gb} / \mathrm{s}(11.2 \mathrm{~Gb} / \mathrm{s}$ net $)$ and $13 \mathrm{~Gb} / \mathrm{s}(10.4 \mathrm{~Gb} / \mathrm{s}$ net) gross information rate, respectively, therefore confirming the potential of such solution for both optical interconnects and access/metro networks scenarios.

The paper is organized as follows: Section II focuses on static and dynamic characterization of the employed VCSEL, including temporal chirp measurement. In Section III the concept of TFP applied to an IM/DD system is explained and the expected performance of the system is investigated through numerical

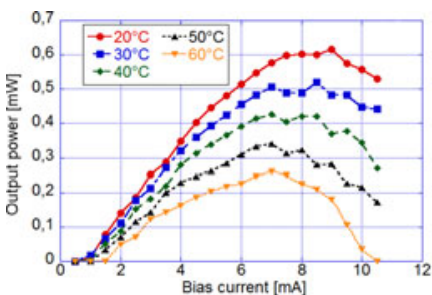

(a)

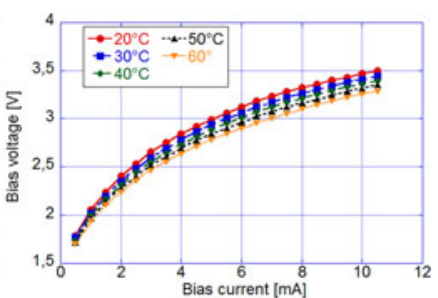

(b)
Fig. 1. Measured optical output power from the VCSEL as a function of the applied bias current (a) and measured bias voltage as a function of the driving bias current (b), both for different operation temperatures.

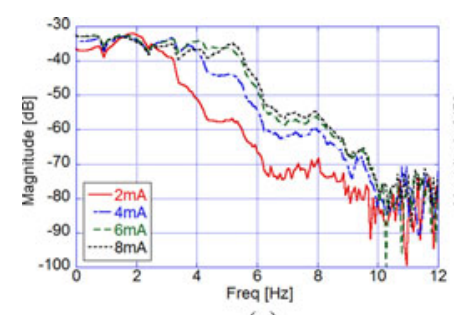

(a)

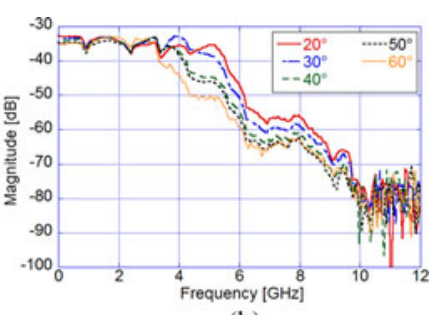

(b)
Fig. 2. Modulation response (S21 magnitude) of the VCSEL at $20{ }^{\circ} \mathrm{C}$ for different bias DC currents (a). S21 magnitude at various operation temperatures where, for each case, the bias current is set so as to maximize the modulation bandwidth (b).

analysis. Section IV describes the transmission experiment and corresponding results, while Section V sums up the conclusions of the work.

\section{DM-VCSEL CHARACTERIZATION}

As a first experimental step, the VCSEL has been fully characterized to investigate its behavior under different operation settings so as to find the optimum working condition. The employed device is a commercial VCSEL supplied by RayCan for $4 \mathrm{~Gb} / \mathrm{s}$ OOK single mode transmission, with emission wavelength of about $1530 \mathrm{~nm}$. The laser exhibits a TOSA package and SMF pig-tail. Optical output power and corresponding bias voltage have been measured as functions of the driving bias direct current (DC) for temperatures ranging from $20^{\circ} \mathrm{C}$ to $60^{\circ} \mathrm{C}$ as shown in Fig. 1. A threshold current of $1 \mathrm{~mA}$ is measured and a roll-over occurs between 7 and $8 \mathrm{~mA}$ where the maximum output power is reached. The maximum achievable optical power decreases as the device temperature increases, ranging from $0.6 \mathrm{~mW}\left(T=20^{\circ} \mathrm{C}\right)$ down to $0.26 \mathrm{~mW}\left(T=60^{\circ} \mathrm{C}\right)$ and the corresponding diode voltage (Fig. 1(b)) leads to typical electrical power consumption below $35 \mathrm{~mW}$.

S21 parameter has been measured through a vector network analyzer (VNA) and a high-speed photodiode. In particular a bias-tee was used to couple the bias DC component and the radio frequency (RF) signal coming from the VNA which was set to a peak-to-peak voltage of $0.2 \mathrm{~V}$. Results are reported in Fig. 2(a) showing, as expected, an increasing small-signal modulation bandwidth as the bias current increases for a fixed operation temperature $\left(20^{\circ} \mathrm{C}\right)$. The $3 \mathrm{~dB}$-bandwidth reaches up to $5 \mathrm{GHz}$ for a bias current of $8 \mathrm{~mA}$. However, several 


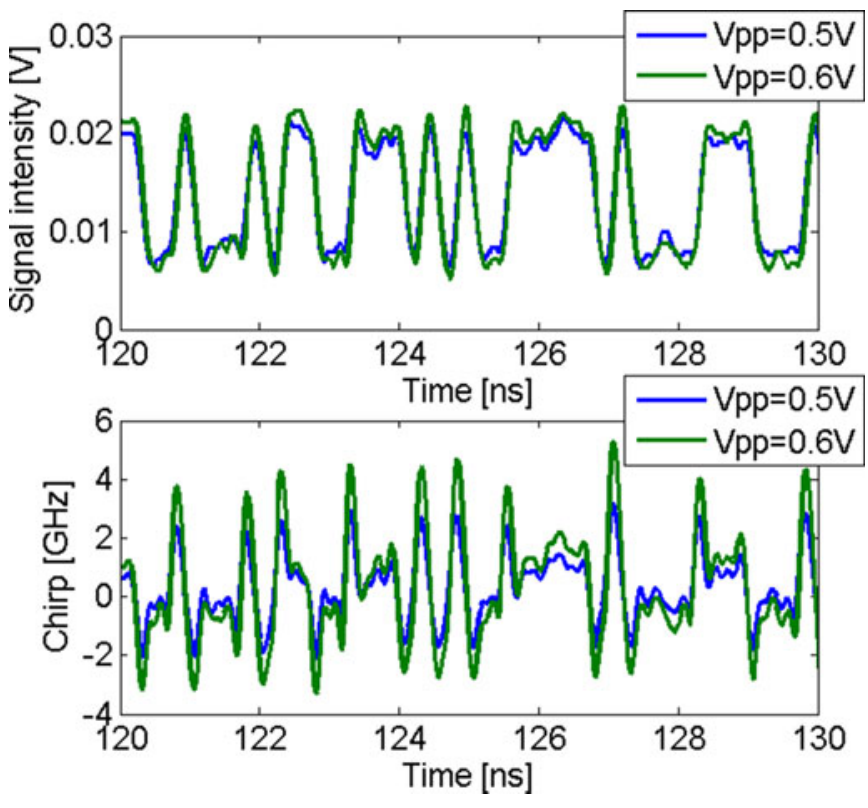

Fig. 3. Temporal intensity of the generated $4 \mathrm{~Gb} / \mathrm{s}$ OOK signal through direct modulation of the VCSEL (up) and resulting temporal frequency chirp measured through the method reported in [24] (down), for a peak-to-peak voltage of the applied data signal equal to $0.5 \mathrm{~V}$ (blue traces) and $0.6 \mathrm{~V}$ (green traces) respectively.

resonance notches appear on the modulation response, possibly due to electric reflections caused by a sub-optimum printed circuit board employed for VCSEL testing. Fig. 2(b) shows how the modulation bandwidth worsens when increasing the temperature from $20{ }^{\circ} \mathrm{C}$ up to $60{ }^{\circ} \mathrm{C}$. In particular, the $3 \mathrm{~dB}$ bandwidth decreases down to $3.9 \mathrm{GHz}$ at $40-50{ }^{\circ} \mathrm{C}$ and to $3.3 \mathrm{GHz}$ at $60{ }^{\circ} \mathrm{C}$.

As stated in Section I, another key feature when making use of DM-VCSELs consists in the dynamic frequency chirp associated to the applied RF modulation current. Indeed, as VCSELs exhibit a cavity length hundreds of times shorter than typical edge-emitting lasers, the needed current density to induce intensity modulation of the output power is very high therefore causing high refractive index changes [23]. The tight power budget caused by such aspect represents the main concern in employing VCSELs for access applications requiring C-band wavelengths and SSMF links. In order to quantify the chirp induced when modulating the VCSEL both in its adiabatic and transient components, the method detailed in [24] has been applied. In particular a $4 \mathrm{~Gb} / \mathrm{s}$ OOK intensity modulation obtained through the DM-VCSEL was injected into an optical differential (D)PSK demodulator that has been employed as the necessary Mach-Zehnder interferometer (MZI), with a relative delay of 23 ps. The tunable phase shifter incorporated in the demodulator served to spectrally shift the MZI response with respect to the signal central wavelength, so as to obtain the three intensity traces required for the calculation of the frequency chirp temporal evolution [24].

The experimental result is shown in Fig. 3 where both the temporal intensity and the temporal frequency chirp are reported for two different peak-to-peak voltages of the applied data signal, namely $0.5 \mathrm{~V}$ and $0.6 \mathrm{~V}$. From the behavior of the instantaneous chirp in Fig. 3 (bottom), it is possible to distinguish both the adiabatic chirp term, that is proportional to the temporal intensity modulation, and its transient term proportional to the derivative of the intensity logarithm, as described in details in [25]. By fitting the chirp model in [25] with the measurements shown in Fig. 3 (bottom), both the enhancement factor $\alpha$ and the adiabatic chirp coefficient $\kappa$ have been extracted and, in particular, $\alpha=3.5$ and 4.7 and $\kappa=0.43 \cdot 10^{12} \mathrm{~Hz} / \mathrm{W}$ and $0.5 \cdot 10^{12} \mathrm{~Hz} / \mathrm{W}$ for a modulation swing of $0.5 \mathrm{~V}$ and $0.6 \mathrm{~V}$ respectively. The average adiabatic term is about $1 \mathrm{GHz}$ for $0.5 \mathrm{~V}$ and $1.9 \mathrm{GHz}$ for $0.6 \mathrm{~V}$, whereas the average transient term reaches $4.3 \mathrm{GHz}$ at $0.5 \mathrm{~V}$ and $6.8 \mathrm{GHz}$ at $0.6 \mathrm{~V}$. On the other hand the intensity extinction ratio (ER) increases from $3.9 \mathrm{~dB}$ to $4.9 \mathrm{~dB}$. Due to the chirp increase and ER increase from $0.5 \mathrm{~V}$ to $0.6 \mathrm{~V}$, a fine optimization of the applied voltage is needed based on this trade-off, as it will be numerically evaluated in next section.

\section{TIME-FREQUENCY PACKING FOR IM/DD SYSTEMS}

The IM/DD TFP system is based on the principles explained in [20], [21]; bandwidth is saved by increasing the signaling rate for a fixed pulse bandwidth (or, equivalently, reducing the pulse bandwidth for a fixed signaling rate). The intentionally induced inter-symbol interference (ISI) is then coped with by a maximum a-posteriori probability (MAP) detector. The benefits can be outlined if we consider the definition of the spectral efficiency

$$
\mathrm{SE}=\frac{I}{F T}[b / s / H z] .
$$

It represents the average mutual information rate per symbol $I$ per unit bandwidth and unit time, being $F T$ the time-frequencyslot devoted to the transmission of each information symbol. ISI has clearly the effect of decreasing $I$, but since $F T$ decreases at the same time, SE can increase, by trading-off between MAP detector complexity and performance.

For further details on TFP and how it relates to former techniques such as its precursor, i.e., Faster-than-Nyquist (or partialresponse signaling, which may resemble TFP, but is based on a completely different approach, purpose, and outcome), please refer to [21]. It is here worth noting that the main difference between TFP and faster than Nyquist (FTN) techniques relies in the system optimization method, which for TFP is based on the maximization of the achievable spectral efficiency, through the exploitation of the principles of auxiliary channel and mismatch detection (see [21] and references therein).

\section{A. Theory}

The proposed IM/DD system entails substantial changes with respect to the already demonstrated optical TFP for coherent detection [21], [22]. It is worth pointing out that the present solution is formally based on the TFP technique, even if in this case a single-channel system is considered, so that the frequency packing concept applies to the transmitted signal but it does not entail the presence of a WDM system. As a matter of fact, the optimization steps are the same, and the presence of 


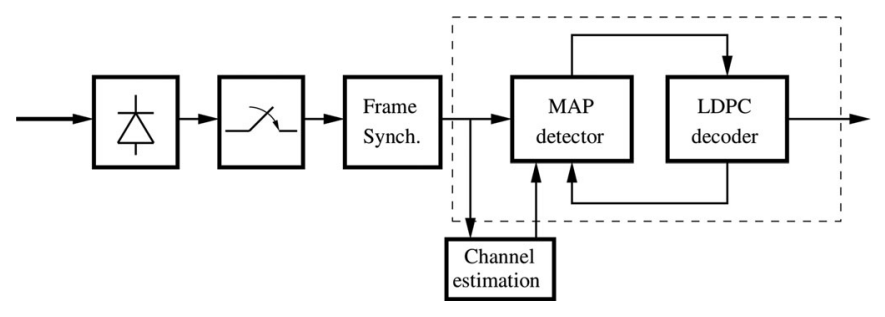

Fig. 4. Schematic of the proposed receiver.

other channels would only affect the final optimized parameters. The receiver side processing is simpler than the coherent system processing as, for instance, frequency synchronization and phase error recovery are not necessary and clock recovery can be combined with the required channel state estimation, even though it is obviously more complex than the processing of a standard IM/DD system; on the other hand the presence of the square-law detector implies a different channel model with respect to the coherent system one. The schematic of the proposed solution is depicted in Fig. 4. The input bit stream is randomly generated and encoded in a sequence $\{c\}$ by using 64800-bit low-density parity check (LDPC) codes in order to assemble a sequence of codewords, each preceded by a known 448-bit pattern (or header) for frame synchronization purposes (see [22] for details on the employed LDPC codes). At the receiver the signal is then sampled at one sample per symbol. The received samples read

$$
r_{k}=\sum_{m=1}^{L} \sum_{n \leq m}^{L} c_{m} c_{n} h(n-k, m-k)+w_{k}
$$

where $w_{k}$ is filtered noise, $h(n, m)$ is the discrete time system response given by a second-order Volterra kernel [26] and $L$ is the channel memory, basically introduced by TFP. Then frame synchronization is performed and afterwards a block of 10k bits is used as a pilot for obtaining an estimate of the $(L+1) L / 2$ coefficients of the system impulse response. Such estimation is performed by exploiting the minimum mean square error (MMSE) algorithm, i.e., by minimizing the function

$$
E\left[r_{k}-\sum_{m}^{L} \sum_{n \leq m}^{L} c_{k-m} c_{k-n} \hat{h}(n, m)\right]^{2},
$$

where $\hat{h}$ denotes estimates. Therefore the update equation for the coefficients reads

$$
\hat{h}^{\ell+1}=\hat{h}^{\ell}+\mu\left(r_{k}-\hat{h}^{\ell} c_{k}\right) c_{k}
$$

where

$$
\begin{aligned}
\hat{h} & =[\hat{h}(1,1), \ldots, \hat{h}(1, L), \hat{h}(2,2), \ldots, \hat{h}(2, L), \ldots, \hat{h}(L, L)], \\
c_{k} & =\left[c_{k} c_{k}, c_{k} c_{k-1}, \ldots, c_{k} c_{k-L}, c_{k-1} c_{k-1}, \ldots, c_{k-L+1} c_{k-L+1}\right],
\end{aligned}
$$

$\mu$ represents a proper step-size and $\ell$ is the update time index.

The impulse response coefficients are then employed by the MAP detector, which accounts for a fixed amount of channel memory $L_{r}$ that can be lower than the actual memory $L$ (in this case entailing a loss of performance). MAP detector iteratively exchanges soft information with the LDPC decoder providing reliable decisions on the transmitted codewords. Results are hence presented both as bit error rate (BER) computations by employing practical LDPC codes and as achievable bit rate lower bounds (ABR), that represents a useful method to evaluate the theoretical system performance without resorting to practical codes. ${ }^{1}$ For insights on the usefulness of such bounds with respect to BER measurements, please refer to [27]. The computation of these bounds exploits indeed the principles of auxiliary channel and mismatched detection, and, in brief, it takes advantage of samples $r_{k}$ to yield a bound $I_{L B} \leq I$, which, despite being a lower bound, is achievable for the considered receiver, in the sense that it is theoretically possible to design a code that reaches the predicted performance.

As already mentioned, the IM/DD system does not require frequency and phase synchronization, whereas ISI handling and clock recovery are included in the channel coefficients estimation for the MAP detector. Hence, the complexity of the proposed receiver lies in the iterative detection/decoding algorithm, which is widely known from literature in wired and wireless communications, and that is becoming increasingly popular in optical systems [28], as the technology progress enables faster and more effective electronic processing.

\section{B. Performance Estimation Through Numerical Analysis}

The DSP receiver based on the concept described in the previous section has been developed. The DSP chain includes all blocks depicted in Fig. 4 next to the analog-to-digital converter (ADC): Frame Synchronization, Channel Estimation and the turbo MAP/LDPC detector.

The expected performance has been analyzed through a numerical simulation in terms of ABR and fiber reach. In particular, a complete VCSEL model has been implemented considering the experimental performance detailed in Section II. Concerning the modulation frequency response, a fitting through the typical 3-pole transfer function for directly-modulated VCSEL [29] of the measured modulation response (S21) at a temperature of $20{ }^{\circ} \mathrm{C}$ (Fig. 2(b)) has been considered. Concerning the frequency chirping model [25], the $\alpha$ and $\kappa$ parameters extracted from measurements in Section II have been considered. The conducted simulation does not take into account the noise at the receiver as, for the APD employed in next section, some of the key noise parameters were not available. Fig. 5 shows the behavior of ABR as a function of the line rate for transmission over $25 \mathrm{~km}$ of SSMF in case of neglecting/including the DM-VCSEL chirp contribution and for peak-to-peak voltage $V_{P P}$ of the applied data equal to $0.5 \mathrm{~V}$ and $0.6 \mathrm{~V}$. In case of no chirp, a higher voltage $(0.6 \mathrm{~V})$ leads to higher ER and higher ABR whose value reaches up to $20.6 \mathrm{~Gb} / \mathrm{s}$. On the other hand, the presence of frequency chirp mixed with the chromatic

\footnotetext{
${ }^{1}$ Notice that we defined achievable bit rate (ABR) lower bounds as opposed to achievable spectral efficiency (ASE) lower bounds, as in [20], because here we are not dealing with a WDM system, hence it would be hard to provide an unequivocal and rigorous definition of bandwidth, which is mandatory to measure the spectral efficiency.
} 


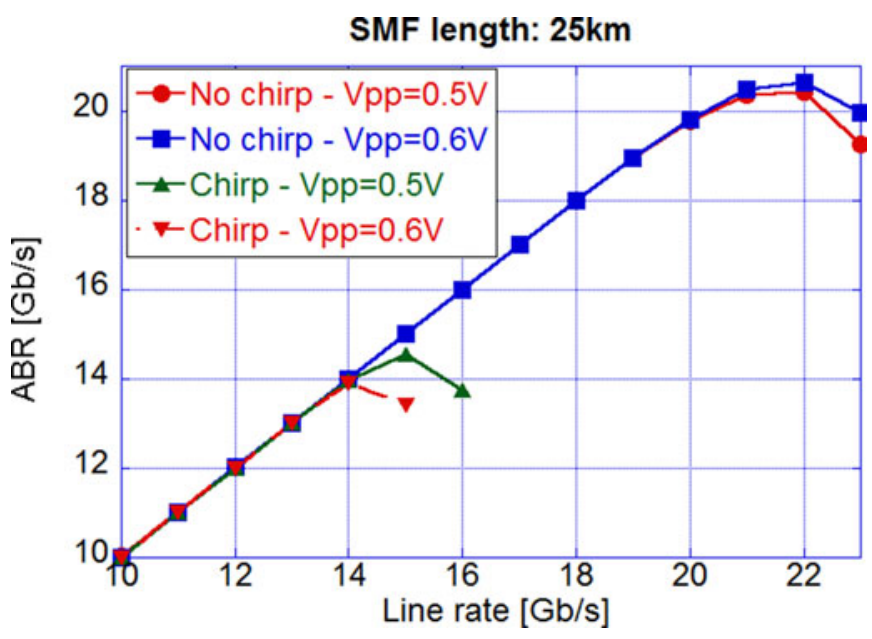

Fig. 5. Simulated achievable bit rate lower bound (ABR) as a function of the line rate in case of $25 \mathrm{~km}$-long SSMF, with and without laser chirp and for peak-to-peak voltage equal to $0.5 \mathrm{~V}$ and $0.6 \mathrm{~V}$.

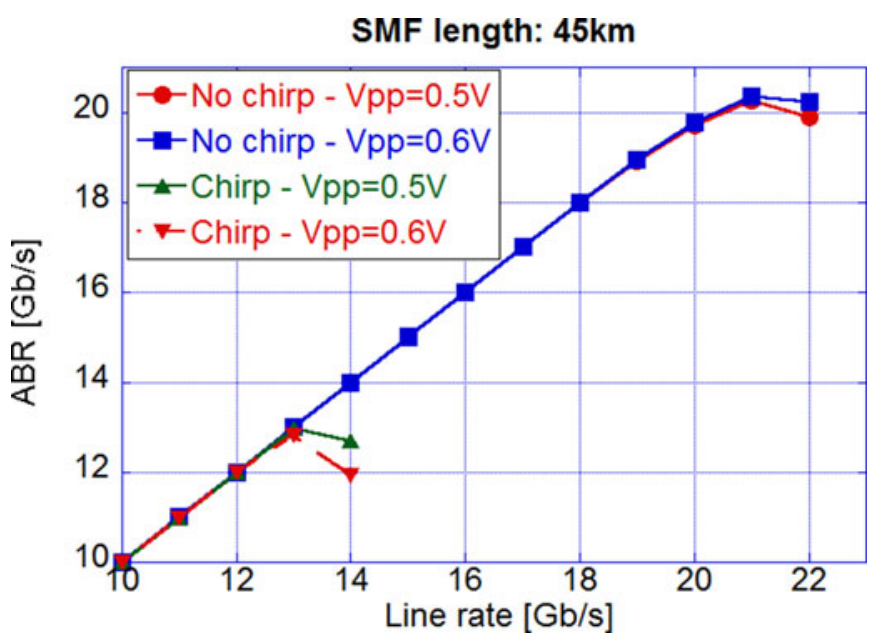

Fig. 6. Simulated achievable bit rate lower bound (ABR) as a function of the line rate in case of $45 \mathrm{~km}$-long SSMF, with and without laser chirp and for peak-to-peak voltage equal to $0.5 \mathrm{~V}$ and $0.6 \mathrm{~V}$.

dispersion (CD) of SSMF in C-band, limits the maximum ABR to $14.5 \mathrm{~Gb} / \mathrm{s}$ and this happens for $V_{P P}=0.5 \mathrm{~V}$ as, in this case, the ABR worsening due to the higher spectral occupation mixed with $\mathrm{CD}$ dominates with respect to the improvement due to a higher ER. Similarly, for $45 \mathrm{~km}$ of SSMF (Fig. 6), the maximum ABR decreases from $20.3 \mathrm{~Gb} / \mathrm{s}$ down to $13 \mathrm{~Gb} / \mathrm{s}$ without and with chirp. The main aim in next section is to experimentally confirm the expected performance in case of chirp, even though an impact of the APD noise is expected to play a role.

\section{EXPERIMENTAL SETUP}

The setup for demonstrating the functionality of the TFP technique for an IM/DD system and for attesting the performance in case of employing a VCSEL as the directly-modulated laser is reported in Fig. 7.

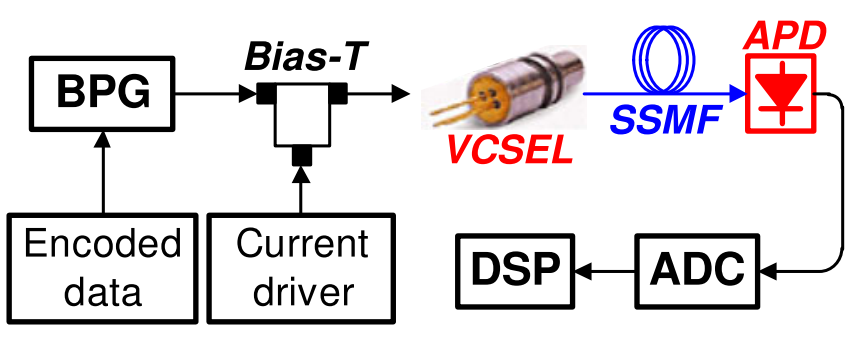

Fig. 7. Experimental setup for the transmission experiments, with electrical/electronic parts in black, optoelectronic devices in red and optical link in blue. BPG: bit pattern generator; ADC: analog-to-digital converter.

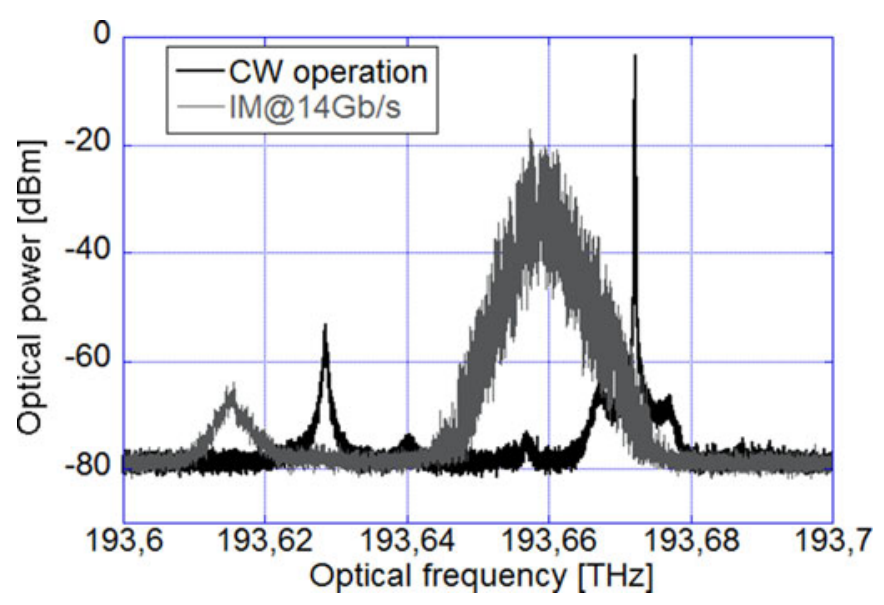

Fig. 8. Optical spectrum in CW operation and for OOK IM at $14 \mathrm{~Gb} / \mathrm{s}$.

At first, the employed VCSEL operates at $20{ }^{\circ} \mathrm{C}$ with $8 \mathrm{~mA}$-bias current, providing an output optical power of $-2.2 \mathrm{dBm}$ with an overall power consumption of $26.5 \mathrm{~mW}$. An additional consumption equal to $13.5 \mathrm{~mW}$ is associated in the particular implementation to thermal stabilization performed by driving the Peltier cell included within the TOSA package through a temperature controller (TEC) that is connected also to the integrated thermistor for monitoring the package temperature. The VCSEL gets directly modulated by a bit pattern generator (BPG) providing low-density parity check (LDPC) encoded data with $0.5 \mathrm{~V}$ peak-to-peak voltage. A bias-tee is employed to combine the RF signal with the bias DC. The VCSEL emission spectrum in continuous wave $(\mathrm{CW})$ operation is visible in Fig. 8, where also the spectrum in case of OOK modulation at $14 \mathrm{~Gb} / \mathrm{s}$ is shown. A side mode suppression ratio $>40 \mathrm{~dB}$ can be observed. After propagation, the received signal is detected through an APD and digitized by a real-time (RT) oscilloscope acting as ADC. In our experiment the RT oscilloscope operates at 25 GSample/s with an analog bandwidth of $5 \mathrm{GHz}$.

Digital data are then re-sampled by the DSP in order to operate at $1 \mathrm{Sample/bit}$. As the target scenario ranges from short-reach optical interconnects to the access and metro segment, at first a $25 \mathrm{~km}$ long SSMF spool was tested and the performance compared with the back-to-back (BtB) case. The DSP unit incorporates the processing described in Section III-A. The ABR is evaluated for different line rates and received optical powers. A channel memory $L_{r}=2$ is chosen to keep the MAP 


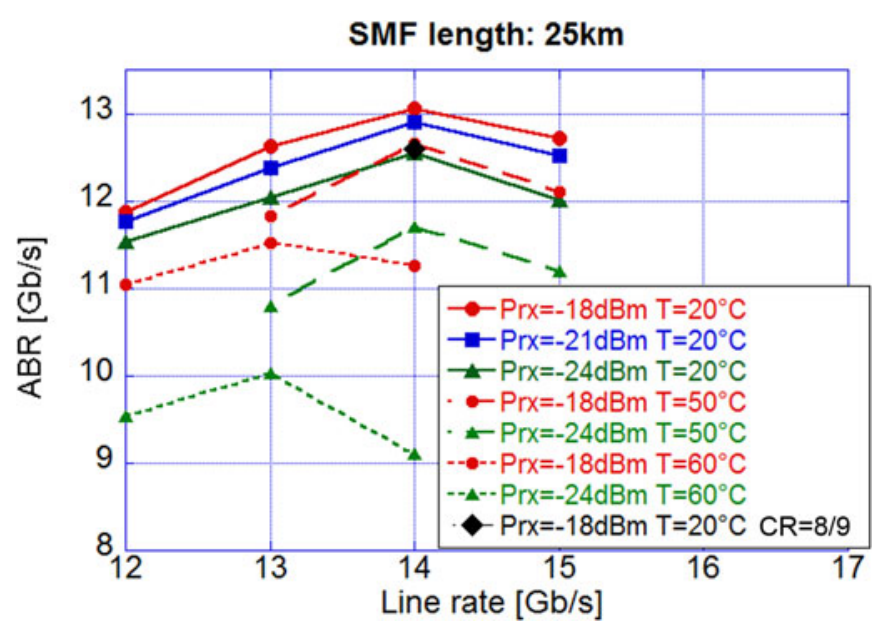

Fig. 9. Achievable bit rate lower bound (ABR) as a function of the line rate in case of $25 \mathrm{~km}$-long SSMF, for different received powers and different temperatures.

detector complexity low, and in order to obtain a good tradeoff between complexity and performance, given the optical components at hand. It is worth reminding that a different detector complexity would have led to a different system optimization. Fig. 9 shows that the maximum $\mathrm{ABR}=13 \mathrm{~Gb} / \mathrm{s}$ is reached for a line rate of $14 \mathrm{~Gb} / \mathrm{s}$ and a received power of $-18 \mathrm{dBm}$. The worse performance with respect to $\mathrm{ABR}=14.5 \mathrm{~Gb} / \mathrm{s}$ estimated in Section III-B is most probably due to the APD noise. In order to demonstrate the suitability of the proposed method at higher temperatures and, therefore, for more relaxed conditions in term of thermal stabilization, the same measurements has been repeated for operation temperature equal to $50{ }^{\circ} \mathrm{C}$ and $60{ }^{\circ} \mathrm{C}$ respectively. The maximum $\mathrm{ABR}$ at $50{ }^{\circ} \mathrm{C}$ is still achieved for a line rate of $14 \mathrm{~Gb} / \mathrm{s}$ and, for a received power of $-18 \mathrm{dBm}$, it is equal to $12.66 \mathrm{~Gb} / \mathrm{s}$. The same measurement repeated for a temperature of $60{ }^{\circ} \mathrm{C}$ indicates, for a received power of $-18 \mathrm{dBm}$, a maximum $\mathrm{ABR}$ of $11.5 \mathrm{~Gb} / \mathrm{s}$ for a line rate equal to $13 \mathrm{~Gb} / \mathrm{s}$. In Fig. 9 it is also reported a result corresponding to the error-free ${ }^{2}$ performance of a rate $8 / 9$ LDPC code in case of received power of $-18 \mathrm{dBm}$ and temperature of $20^{\circ} \mathrm{C}$, which confirms that proper codes can approach the lower bounds with good accuracy.

Concerning actual bit error rate (BER) measurements, for a temperature of $20{ }^{\circ} \mathrm{C}$ error-free operation is demonstrated by employing a LDPC code with a rate $R_{c}=8 / 9$ corresponding to a net bit rate of $12.5 \mathrm{~Gb} / \mathrm{s}$ with a consequent energy consumption per bit of $2.13 \mathrm{pJ} / \mathrm{bit}$ (net of the $1.1 \mathrm{pJ} / \mathrm{bit}$ needful for thermal stabilization). Comparative measures showing the required turbo decoder iterations versus received optical power are performed. Results for transmission up to $25 \mathrm{~km}$ and $12.5 \mathrm{~km}$ of SSMF and in the BtB case are depicted in Fig. 10, showing a power budget of about $19 \mathrm{~dB}$ and $21 \mathrm{~dB}$ for $25 \mathrm{~km}$ and $12.5 \mathrm{~km}$ respectively. Similarly, the transmission performance

\footnotetext{
${ }^{2}$ Error-free actually refers to a BER of $10^{-6}$, which can be approximately considered a threshold for the decoding algorithm convergence, since it is not possible to measure lower BERs in a reasonable amount of time.
}

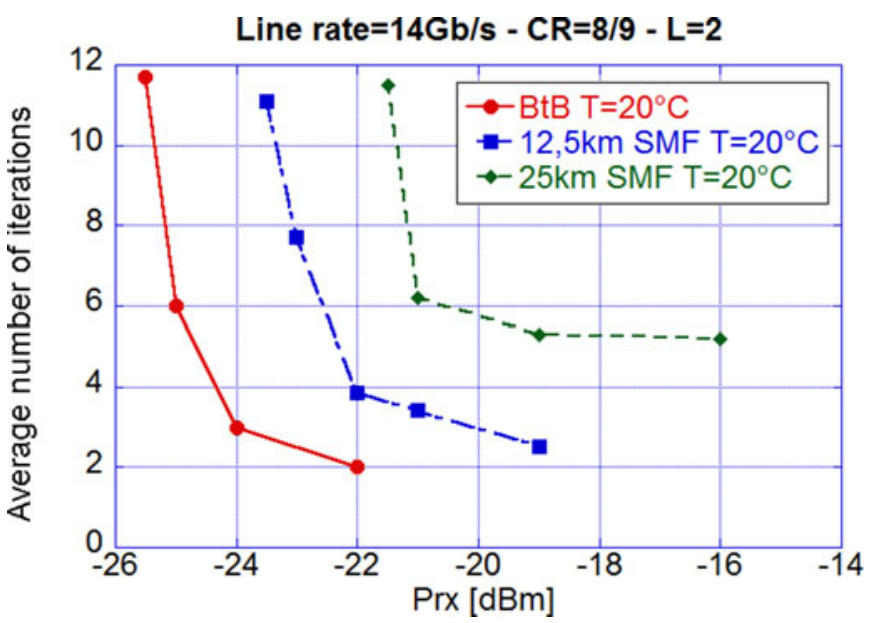

Fig. 10. Average turbo decoder iterations versus received power for a line rate of $14 \mathrm{~Gb} / \mathrm{s}$, code rate $8 / 9$ and different propagation lengths $\left(T=20{ }^{\circ} \mathrm{C}\right)$.

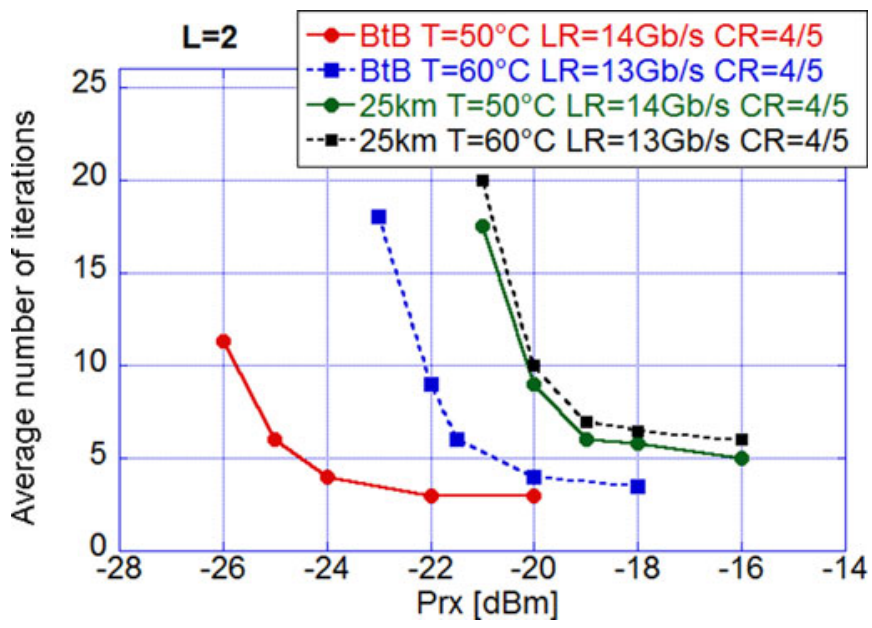

Fig. 11. Average turbo decoder iterations versus received power for temperatures of $50{ }^{\circ} \mathrm{C}$ and $60^{\circ} \mathrm{C}$. In both cases the measurement has been performed for a $25 \mathrm{~km}$-long SSMF in comparison with the back-to-back case. Line and code rates are reported in the legend for each curve.

has been tested for temperatures of $50{ }^{\circ} \mathrm{C}$ and $60^{\circ} \mathrm{C}$, comparing the transmission over $25 \mathrm{~km}$ of $\mathrm{SMF}$ with the BtB case. Fig. 11 shows that, with respect to the $20{ }^{\circ} \mathrm{C}$ case, at $50{ }^{\circ} \mathrm{C}$ error-free operation is demonstrated by lowering the LDPC code rate to $4 / 5$, corresponding to a net bit rate of $11.2 \mathrm{~Gb} / \mathrm{s}$ and leading to a power budget of $19 \mathrm{~dB}$. Heating up the laser to $60^{\circ} \mathrm{C}$, error-free operation was possible lowering both the code rate to $4 / 5$ and the line rate to $13 \mathrm{~Gb} / \mathrm{s}$, leading to a net information rate of $10.4 \mathrm{~Gb} / \mathrm{s}$ with a power budget of again $19 \mathrm{~dB}$. For higher temperatures the laser has proven to survive even though no long-term tests were conducted, but the system performance dropped such that Authors did not consider relevant to conduct further detailed transmission experiments.

As additional measure, the ABR for a longer distance of $45 \mathrm{~km}$ was measured and this was possible for a temperature of $20^{\circ} \mathrm{C}$ only. The maximum $\mathrm{ABR}=11.2 \mathrm{~Gb} / \mathrm{s}$ is reached for a line rate of $11.5 \mathrm{~Gb} / \mathrm{s}$ and a received power of $-18 \mathrm{dBm}$ 


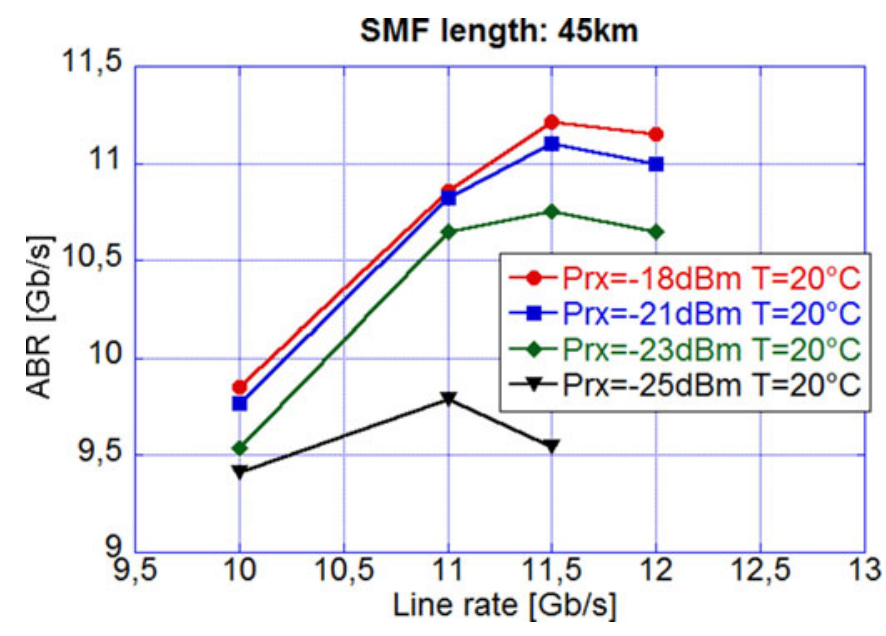

Fig. 12. ABR versus line rate in case of $45 \mathrm{~km}$ of SSMF, for different received powers $\left(T=20^{\circ} \mathrm{C}\right)$.

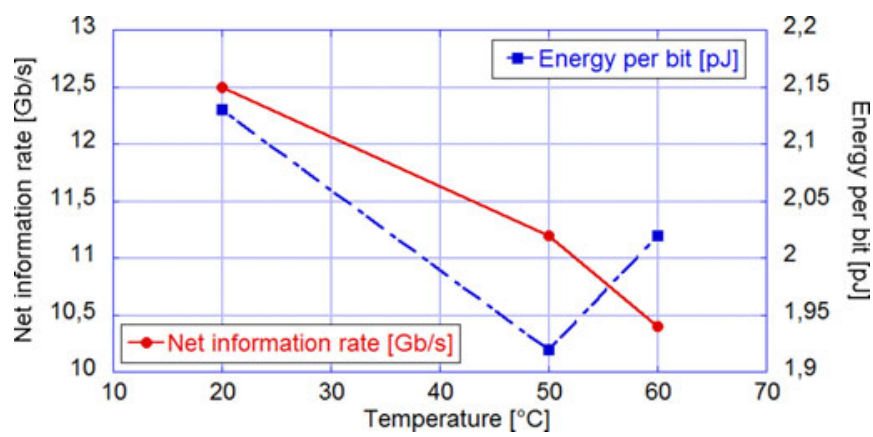

Fig. 13. Summary of the achieved net information rate and energy consumption per bit, versus the considered operation temperature, in case of transmission up to $25 \mathrm{~km}$ of SSMF.

(Fig. 12). Even in this case the discrepancy from the performance estimated through numeric simulation reported by Section III-B $(\mathrm{ABR}=13 \mathrm{~Gb} / \mathrm{s})$ is most probably due to the impact of the employed APD noise. In this case error-free operation is obtained with a code rate $R_{c}=8 / 9$ corresponding to a net bit rate of $10.2 \mathrm{~Gb} / \mathrm{s}$. It can be noticed that for a received power of $-25 \mathrm{dBm}$, the maximum $\mathrm{ABR}$ is located at $11 \mathrm{~Gb} / \mathrm{s}$ rather than $11.5 \mathrm{~Gb} / \mathrm{s}$, as for other received powers, since in this case the signal is primarily limited by a low SNR rather than ISI.

Fig. 13 sums up the obtained results for successful transmission up to $25 \mathrm{~km}$ of SSMF, considering an uncooled scenario where the transceiver temperature is free to reach up to $60{ }^{\circ} \mathrm{C}$. Therefore the plotted energy consumption per bit is referred to the transmitter only and, since a temperature controller was employed in the particular implementation with the only purpose of stabilizing the operation temperature, any thermal management is considered in such calculation. Despite the decrease of the net information rate from $20^{\circ} \mathrm{C}$ to $50{ }^{\circ} \mathrm{C}$, the energy per bit decrease is due to the bias voltage decrease (see Fig. 1(b)). Then, by increasing more the temperature up to $60^{\circ} \mathrm{C}$, the lowering of the net information rate dominates the bias voltage decrease so as to cause higher energy consumption.

\section{CONCLUSION}

A polarization- and wavelength-independent intensity modulation/direct detection (IM/DD) transmission system has been proposed and experimentally validated by using a low-cost commercial 4G directly-modulated (DM)-VCSEL at the transmitter and an avalanche photodiode at the receiver. Thanks to the timefrequency packing (TFP) technique, adapted and employed here in an IM/DD system for the first time, a net bit rate $(12.5 \mathrm{~Gb} / \mathrm{s})$ three times higher than the employed VCSEL nominal speed is experimentally achieved and successfully transmitted over $25 \mathrm{~km}$ of SSMF with a $19 \mathrm{~dB}$ power budget and energy consumption of $3.4 \mathrm{pJ} / \mathrm{bit}$ including thermal management. A net bit rate of $10.2 \mathrm{~Gb} / \mathrm{s}$ is achieved for $45 \mathrm{~km}$ of SSMF with a $16-\mathrm{dB}$ power budget. TFP is performed at the receiver by a lowcomplexity DSP $\left(\mathrm{L}_{\mathrm{r}}=2\right)$ unit, whereas the narrow-filtering of the $14 \mathrm{~Gb} / \mathrm{s}$ (gross rate) electrical signal is implemented at the transmitter by the limited VCSEL bandwidth. The system does not require chromatic dispersion compensation, optical amplification, coherent detection and DSP at the transmitter. The low complexity and energy consumption of the system, together with the potential low cost, make the proposed solution a good candidate for next-generation WDM access and metro network applications.

\section{ACKNOWLEDGMENT}

The authors would like to thank Marco Presi for technical support and fruitful scientific discussion on DM-VCSEL.

\section{REFERENCES}

[1] FP7 European Project RAPIdO (G.A. n. 619806), 2014. [Online]. Available: http://www.rapido-project.eu

[2] F. Koyama, "VCSEL integration for silicon photonics," in Proc. 3rd IEEE Int. Conf. Group IV Photon., Ottawa, ON, Canada, 2006, pp. 194-196.

[3] T. Aalto et al., "Multi-wavelength transceiver integration on SOI for high-performance computing system applications," Proc. SPIE, vol. 9368, 2015, Art. no. 93680B.

[4] T. Aalto et al., "Integrating III-V, $\mathrm{Si}$, and polymer waveguides for optical interconnects: RAPIDO," (Invited Paper), in Proc. SPIE, Opt. Interconnects XVI, Vol. 9753, Feb. 15-17, 2016, Art. no. 97530D.

[5] M. Ortsiefer et al., "Long wavelength high speed VCSELs for long haul and data centers," in Proc. Opt. Fiber Commun. Conf. Exhib., San Francisco, CA, USA, 2014, Paper W4C.2.

[6] 2007. [Online]. Available: http://www.vertilas.com/sites/default/files/ Downloads/vertilas_communications_v5_0_0.pdf

[7] 2012. [Online]. Available: http://www.bandwidth10.com/bw10-1550-10t-s_v1.pdf

[8] A. Malacarne et al., "Performance analysis of $40 \mathrm{~Gb} / \mathrm{s}$ transmission based on directly modulated high-speed 1530-nm VCSEL," IEEE Photon. Technol. Lett., vol. 28, no. 16, pp. 1735-1738, Aug. 2016.

[9] F. Karinou, R. Rodes, K. Prince, I. Roudas, and I. T. Monroy, "IM/DD vs. 4-PAM using a 1550-nm VCSEL over short-range SMF/MMF links for optical interconnects," in Proc. Opt. Fiber Commun. Conf. Exp. Nat. Fiber Opt. Eng. Conf., Anaheim, CA, USA, 2013, Paper OW4A.2.

[10] R. Rodes et al., "High-speed $1550 \mathrm{~nm}$ VCSEL data transmission link employing 25 GBd 4-PAM modulation and hard decision forward error correction," J. Lightw. Technol., vol. 31, no. 4, pp. 689-695, Feb. 2013.

[11] B. Zhang et al., "Adjustable chirp injection-locked 1.55- $\mu \mathrm{m}$ VCSELs for enhanced chromatic dispersion compensation at 10-Gbit/s," in Proc. Opt. Fiber Commun. Nat. Fiber Opt. Eng. Conf., San Diego, CA, USA, 2008, Paper OWT7.

[12] A. Gatto, A. Boletti, P. Boffi, and M. Martinelli, "Adjustablechirp VCSEL-to-VCSEL injection locking for $10-\mathrm{Gb} / \mathrm{s}$ transmission at $1.55 \mu \mathrm{m}, " O p t$. Express, vol. 17, no. 24, pp. 21748-21753, 2009. 
[13] J. Zhou, C. Yu, and H. Kim, "1.5- $\mu \mathrm{m}, 21.4-G b p s$ 4-PAM VCSEL link for optical access applications," in Proc. Opt. Fiber Commun. Conf. Exhib., Los Angeles, CA, USA, 2015, Paper Th2A.54.

[14] G. Meloni, A. Malacarne, F. Fresi, and L. Potì, " 6.27 bit/s/Hz spectral efficiency VCSEL-based coherent communication over 800km of SMF," in Proc. Opt. Fiber Commun. Conf. Exhib., Los Angeles, CA, USA, Mar. 2015, Paper Th2A.30.

[15] T. N. Huynh et al., "Digital coherent communications with a $1550 \mathrm{~nm}$ VCSEL," in Proc. Opt. Fiber Commun. Conf. Exhib., Los Angeles, CA, USA, 2015, Paper M2D.7.

[16] C. Xie et al., "960-km SSMF transmission of 105.7-Gb/s PDM 3-PAM using directly modulated VCSELs and coherent detection," Opt. Express, vol. 21, no. 9, pp. 11585-11589, 2013.

[17] C. Xie et al., "Generation and transmission of a 400-Gb/s PDM/WDM signal using a monolithic $2 \times 4$ VCSEL array and coherent detection," in Proc. Opt. Fiber Commun. Conf. Exhib., San Francisco, CA, USA, 2014, Paper Th3K.2.

[18] R. Corsini, M. Presi, M. Artiglia, and E. Ciaramella, "10-Gb/s long-reach PON system with low-complexity dispersion-managed coherent receiver," IEEE Photon. J., vol. 7, no. 5, Oct. 2015, Art. no. 7103008.

[19] A. Malacarne, F. Fresi, G. Meloni, T. Foggi, and L. Potí, "Time-frequency packed VCSEL-based IM/DD transmission for WDM access networks," in Proc. 2016 21st OptoElectron. Commun. Conf., Niigata, Japan, 2016, Paper MF1-5.

[20] A. Barbieri, D. Fertonani, and G. Colavolpe, "Time-frequency packing for linear modulations: Spectral efficiency and practical detection schemes," IEEE Trans. Commun., vol. 57, no. 10, pp. 2951-2959, Oct. 2009.

[21] G. Colavolpe and T. Foggi, "Time-frequency packing for high capacity coherent optical links," IEEE Trans. Commun., vol. 62, no. 8, pp. 29862995, Aug. 2014.
[22] M. Secondini et al., "Optical time-frequency packing: Principles, design, implementation, and experimental demonstration," J. Lightw. Technol., vol. 33, no. 17, pp. 3558-3570, Sep. 2015.

[23] C. J. Chang-Hasnain, "VCSEL for metro communications," in Optical Fiber Telecommunications IV-A, 4th ed. New York, NY, USA: Academic, 2002, ch. 13, sec. 2, pp. 668-670.

[24] C. Laverdiere, A. Fekecs, and M. Tetu, "A new method for measuring time-resolved frequency chirp of high bit rate sources," IEEE Photon. Technol. Lett., vol. 15, no. 3, pp. 446-448, Mar. 2003.

[25] G. P. Agrawal and N. K. Dutta, Long-Wavelength Semiconductor Lasers. New York, NY, USA: Van Nostrand Reinhold, 1993.

[26] W. Chung, "Channel estimation methods based on volterra kernels for MLSD in optical communication systems," IEEE Photon. Technol. Lett., vol. 22, no. 4, pp. 224-226, Feb. 2010.

[27] T. Foggi, G. Colavolpe, A. Bononi, and P. Serena, "Spectral efficiency optimization in flexi-grid long-haul optical systems," J. Lightw. Technol., vol. 33, no. 13, pp. 2735-2742, Jul. 2015.

[28] I. B. Djordjevic and T. Wang, "Multiple component codes based on generalized LDPC code for high-speed optical transport," Opt. Express, vol. 22, no. 4, pp. 16694-16705, 2014

[29] M. Muller et al., "1550-nm high-speed short-cavity VCSELs," IEEE J. Sel. Topics Quantum Electron., vol. 17, no. 5, pp. 1158-1166, Sep./Oct. 2011

Authors' biographies not available at the time of publication. 